\title{
Phase-Sensitive OTDR Strain Measurement Using Single Mode Operation in Multimode OM4 Fiber \\ Krzysztof Markiewicz, ${ }^{1,2 *}$, Jakub Kaczorowski, ${ }^{1,2}$, Zhisheng Yang ${ }^{1}$, Lukasz Szostkiewicz ${ }^{2,3}$, Alejandro Dominguez-Lopez ${ }^{2}$, Krzysztof Wilczyński ${ }^{4}$, Marek Napierala ${ }^{2}$, Tomasz Nasitowski², Luc Thévenaz ${ }^{1}$
}

\author{
${ }^{1}$ EPFL Swiss Federal Institute of Technology, Institute of Electrical Engineering, SCI STI LT, Station 11, CH- \\ 1015 Lausanne, Switzerland \\ ${ }^{2}$ InPhoTech Sp. z o. o., 12 Dzika st 15 Warsaw, 00-195, Poland \\ ${ }^{3}$ Faculty of Physics, Warsaw University of Technology, Warsaw 00-662, Poland \\ ${ }^{4}$ Polish Centre For Photonics And Fibre Optics, 312 Rogoznica, 36-060 Glogow Malopolski, Poland \\ *kmarkiewicz@inphotech.pl
}

Keywords: Optical fiber sensors, Multimode fiber, Strain sensing, Phase-sensitive Optical Time-Domain Reflectometry, Spatial division multiplexing

We present the first realization of strain sensing in a multimode OM4 optical fiber using phase-sensitive optical time-domain reflectometry. We demonstrate that single mode operation in an OM4 fiber is achievable and allows the fiber strain to be measured in a distributed manner. The $4 \mathrm{~km}$ of OM4 fiber is effectively measured with $20 \mathrm{~cm}$ spatial resolution which proves that coherent Rayleigh scattering based techniques can be used to interrogate multimode optical fibers.

\begin{abstract}
\section{Introduction}

Over the past few decades distributed fiber optic sensors based on Rayleigh, Brillouin and Raman scattering have been widely studied due to their possible application in measuring physical parameters like strain, temperature or vibrations in a continuous manner along tens of kilometers with precise identification of the location of the event [1-6]. This research has been mainly focused on single mode fibers due to the lack of intermodal effects. On the other hand, space division multiplexing (SDM) techniques have recently been investigated for telecommunication applications so selective mode management has turned into a rapidly developing technique [7-9]. Few- and multi-mode fibers are among the most promising solutions to replace single-mode fibers for telecom, and it can be expected that long distance multi- and few-mode links will be deployed in a foreseeable future. Since distributed optical fiber sensing is often used in existing fiber networks and Raman- and Brillouin-based techniques already find themselves applicable in multi-mode media [10-14], we investigate the possibility to utilize multimode links to perform phase-sensitive optical time-domain reflectometry ( $\phi$-OTDR). Although the possibility to use multimode fibers was presented earlier for acoustic sensing $[\mathbf{1 5 , 1 6}]$, the proposed techniques did not facilitate single-mode operation in OM4 fiber. In this paper, we present the first direct $\phi$-OTDR strain measurement using single-mode operation in a $4 \mathrm{~km}$ multi-mode OM4 optical fiber with $20 \mathrm{~cm}$ spatial resolution.
\end{abstract}

\section{Methodology}

To investigate the OM4 fiber response to strain we developed a high-resolution $\phi$-OTDR setup, analog to the one depicted in [17], but combined with a modal filter that ensured efficient single-mode excitation. As a light source, we employed a DFB laser operating at a central wavelength of $1550 \mathrm{~nm}$ and with a tuning range of $30 \mathrm{GHz}$. A synchronized electro-optic modulator (EOM) and a semiconductor optical amplifier (SOA) were used to generate $2 \mathrm{~ns}$ pulses with sufficient extinction ratio to enable measurements with $20 \mathrm{~cm}$ of spatial resolution over $4 \mathrm{~km}$ range. In addition, two erbium-doped fiber amplifiers (EDFA) were utilized. The first one to boost the pulse power and the second one to amplify the returning scattered signal before detection. To ensure single-mode operation, the all-fiber mode filter, fabricated by InPhoTech, as depicted in [18], was placed at the input of the fiber under test. The mode filter works bi-directionally, ensuring an insertion loss for the fundamental mode of $\sim 2 \mathrm{~dB}$. To confirm that the filter secures a single-mode operation, a beam quality factor $\mathrm{M}^{2}=1.06$ was measured at the fiber far end. At the last stage before signal detection, a tunable filter (of $1 \mathrm{~nm}$ bandwidth) was placed, in order to filter the out-of-band noise from the second EDFA. Ultimately, a DC-coupled photodiode

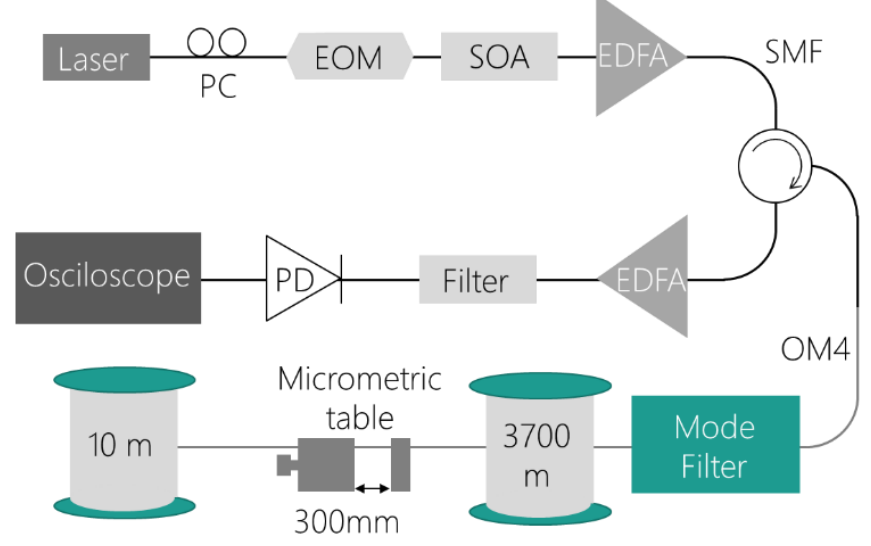

Figure 1 Experimental setup used for strain measurements in OM4 fiber with $\phi$-OTDR. PC - polarization controller, EOM - electro-optic modulator, SOA - semiconductor optical amplifier, EDFA - Erbium Doped Fiber Amplifier, PD - photodiode, SMF - single mode fiber, OM4- multimode OM4 optical fiber 
was utilized at detection, featuring a bandwidth of $1 \mathrm{GHz}$, which is sufficient to properly retrieve the target spatial resolution. Then, the electrical signal from the detector is digitized by means of a $4 \mathrm{GHz}$ bandwidth oscilloscope.

To induce strain in a controllable way over the optical fiber we fixed the fiber to a micrometric translation stage. Strain measurements were carried out by comparing two frequency scans, measured before and after inducing the strain [17]. For each point of the fiber, the cross-correlation of intensity versus frequency data for the two scans was calculated. Based on the maximum of the calculated cross-correlation, a frequency shift is retrieved, which is a linear function of the induced strain.

\section{Results}

As a first step, we verified that the obtained trace for a multimode fiber has sufficient quality to perform the measurement. We checked that each visible peak in the measured patterns presents a full-width at half-maximum (FWHM) of $20 \mathrm{~cm}$, which confirms that the target spatial resolution is nearly equivalent [19]. As a confirmation, we calculated the visibility of the peaks, defined as $\frac{I_{\max }-I_{\min }}{\boldsymbol{I}_{\max }+I_{\min }}$ over a 10 meter region (where $I_{\max }$ and $I_{\min }$ are respectively the maximum and the minimum intensity of the trace). For each segment calculated, the value is over 0.8 , which gives good confidence in the resolving power of the system. An example of a visible trace (blue line) and a calculated visibility (green line) is shown on Figure 2.

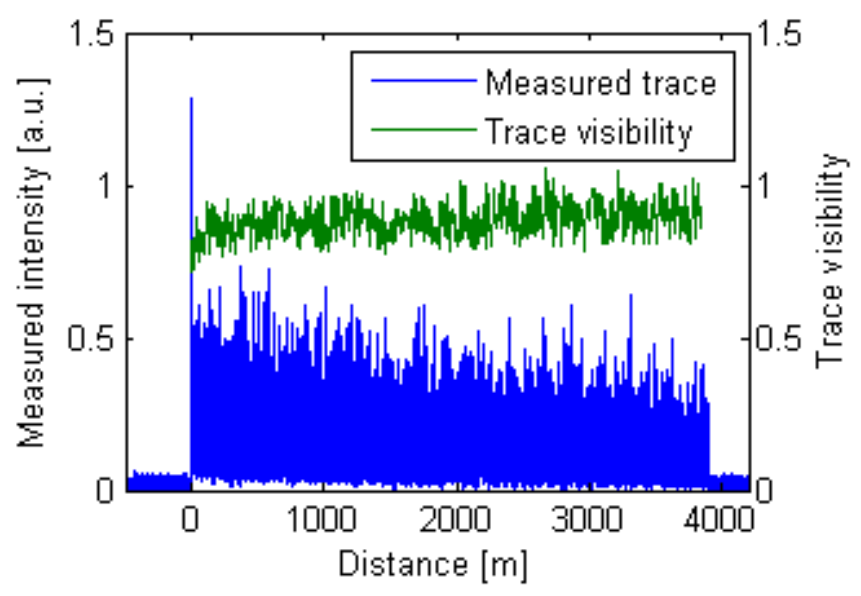

Figure 2 Measured trace (blue line) and calculated visibility of pattern over $10 \mathrm{~m}$ segments. (green line)

As a second step, we examined the frequency shift induced by the change in strain on the last few meters of the optical fiber. We measured the frequency pattern for three different applied strains. Each time we measured the pattern twice to ensure that the pattern is stable if no additional strain is applied. On Figure 3 , the calculated cross-correlation is shown as a function of the frequency shift for every point along the fiber. On the upper graph, it is visible that the cross-correlation has a maximum for zero frequency shift for all points along the fiber except for the part where the strain is applied. The strained part is visible on the lower graph in Fig 3, which is an enlarged part of the upper graph. The impact of the applied strain is clearly visible as a shift in the maximum value of the cross-correlation. By measuring the frequency shift for three different strains and fitting a linear function we determined the sensitivity of the fundamental mode in the OM4 optical fiber to be $138 \mathrm{MHz} / \mu \varepsilon$.

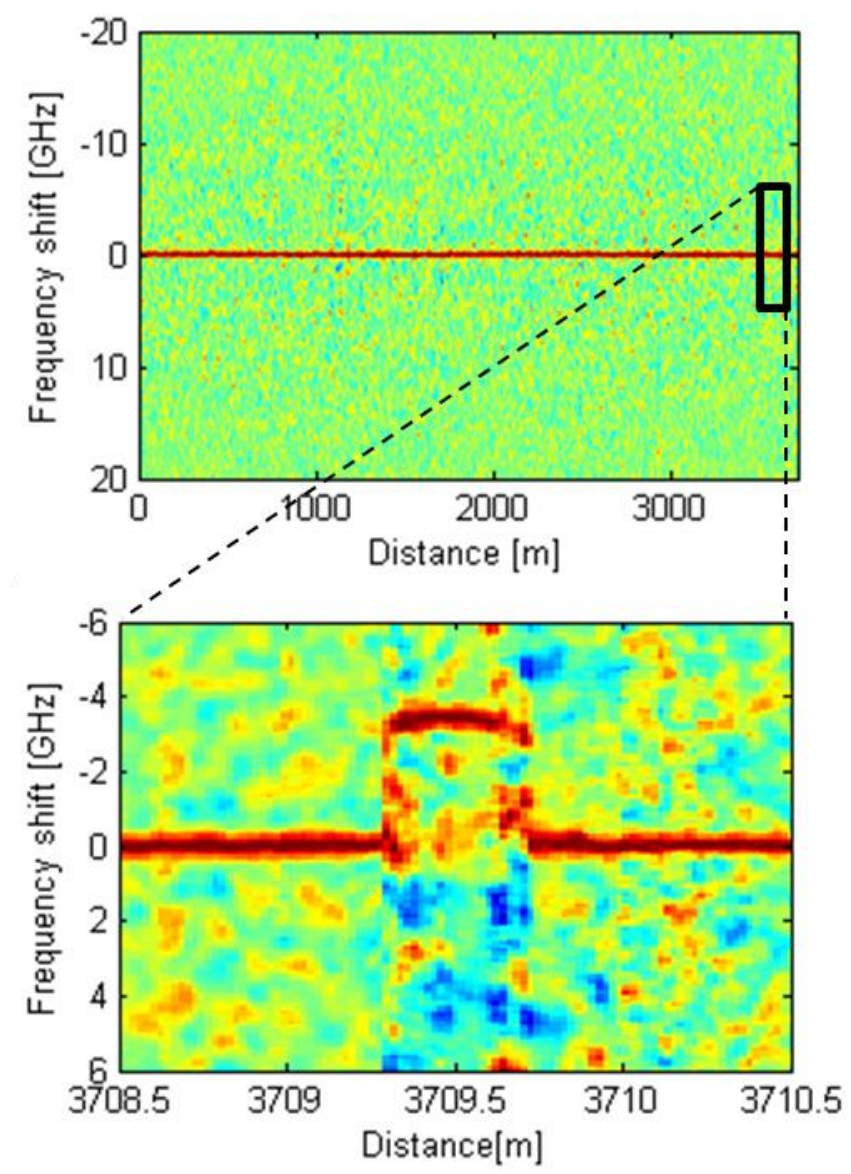

Figure 3 Calculated cross-correlation in in function of frequency shift for each point if optical fiber. Lower graph is zoomed part of the section where strain was induced.

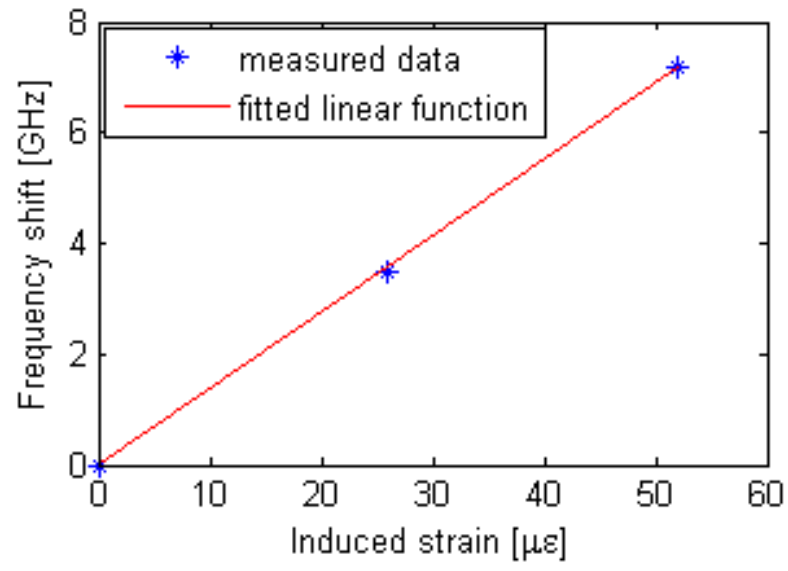

Figure 4 Measured frequency shift as a function of the induced strain together with the fitted linear function. The determined strain sensitivity, for the fundamental mode of the OM4 optical fiber, based on the linear fitting is equal to $138 \mathrm{MHz} / \mu \varepsilon$. 


\section{Conclusion}

To the best of our knowledge we have demonstrated the first strain measurement with a $\phi$-OTDR setup, using single-mode operation in a multi-mode fiber. The measured strain sensitivity of the fundamental mode in an OM4 fiber is proven to be $138 \mathrm{MHz} / \mu \varepsilon$, which is lower than value for standard telecom single mode fiber equal to $150 \mathrm{MHz} / \mu \varepsilon$ [6]. These results prove that it is possible to perform $\phi$-OTDR measurements over multi-mode links, hence, enabling the inspection of already deployed networks in a detailed manner.

\section{Acknowledgements}

The project leading to this application has received funding within the "NODUS" project carried out within the TEAMTECH programme of the Foundation for Polish Science co-financed by the European Union under the European Regional Development Fund and was also supported by the National Centre for Research and Development within the research

TECHMATSTRATEG1/348438/16/NCBR/2018

\section{References}

1. J. C. Juarez, E. W. Maier, Kyoo Nam Choi, and H. F. Taylor, "Distributed fiber-optic intrusion sensor system," J. Light. Technol. 23, 2081-2087 (2005).

2. A. F. Fernandez, P. Rodeghiero, B. Brichard, F. Berghmans, A. H. Hartog, P. Hughes, K. Williams, and A. P. Leach, "Radiation-tolerant Raman distributed temperature monitoring system for large nuclear infrastructures," IEEE Trans. Nucl. Sci. 52, 2689-2694 (2005).

3. J. M. Lopez-Higuera, L. Rodriguez Cobo, A. Quintela Incera, and A. Cobo, "Fiber Optic Sensors in Structural Health Monitoring," J. Light. Technol. 29, 587-608 (2011).

4. C. K. Kirkendall and A. Dandridge, "Overview of high performance fibre-optic sensing," J. Phys. Appl. Phys. 37, R197-R216 (2004).

5. A. Masoudi and T. P. Newson, "Contributed Review: Distributed optical fibre dynamic strain sensing," Rev. Sci. Instrum. 87, 011501 (2016).

6. X. Lu, M. A. Soto, and L. Thévenaz, "Temperaturestrain discrimination in distributed optical fiber sensing using phase-sensitive optical time-domain reflectometry," Opt. Express 25, 16059 (2017).

7. M. Jinno, "Spatial Channel Network (SCN): Opportunities and Challenges of Introducing Spatial Bypass Toward the Massive SDM Era [Invited]," J. Opt. Commun. Netw. 11, 1 (2019).

8. P. J. Winzer, D. T. Neilson, and A. R. Chraplyvy, "Fiber-optic transmission and networking: the previous 20 and the next 20 years [Invited]," Opt. Express 26, 24190 (2018).

9. W. Klaus, B. J. Puttnam, R. S. Luís, J. Sakaguchi, J.M. D. Mendinueta, Y. Awaji, and N. Wada, "Advanced Space Division Multiplexing Technologies for Optical Networks," J. Opt. Commun. Netw. 9, C1 (2017).
10. M. Wang, H. Wu, M. Tang, Z. Zhao, Y. Dang, C. Zhao, R. Liao, W. Chen, S. Fu, C. Yang, W. Tong, P. P. Shum, and D. Liu, "Few-mode fiber based Raman distributed temperature sensing," Opt. Express 25, 4907 (2017).

11. H. Wu, R. Wang, D. Liu, S. Fu, C. Zhao, H. Wei, W. Tong, P. P. Shum, and M. Tang, "Few-mode fiber based distributed curvature sensor through quasisingle-mode Brillouin frequency shift," Opt. Lett. 41, 1514 (2016).

12. H. Wu, M. Tang, M. Wang, C. Zhao, Z. Zhao, R. Wang, R. Liao, S. Fu, C. Yang, W. Tong, P. P. Shum, and D. Liu, "Few-mode optical fiber based simultaneously distributed curvature and temperature sensing," Opt. Express 25, 12722 (2017).

13. P. Xu, Y. Dong, J. Zhang, D. Zhou, T. Jiang, J. Xu, H. Zhang, T. Zhu, Z. Lu, L. Chen, and X. Bao, "Bendinsensitive distributed sensing in singlemodemultimode-singlemode optical fiber structure by using Brillouin optical time-domain analysis," Opt. Express 23, 22714-22722 (2015).

14. Y. Weng, E. Ip, Z. Pan, and T. Wang, "Single-end simultaneous temperature and strain sensing techniques based on Brillouin optical time domain reflectometry in few-mode fibers," Opt. Express 23, 9024 (2015).

15. A. E. Alekseev, V. S. Vdovenko, B. G. Gorshkov, V. T. Potapov, and D. E. Simikin, "Fading reduction in a phase optical time-domain reflectometer with multimode sensitive fiber," Laser Phys. 26, 095101 (2016).

16. M. J. Murray, A. Davis, and B. Redding, "Multimode fiber $\Phi-O T D R$ with holographic demodulation," Opt. Express 26, 23019 (2018).

17. Y. Koyamada, M. Imahama, K. Kubota, and K. Hogari, "Fiber-Optic Distributed Strain and Temperature Sensing With Very High Measurand Resolution Over Long Range Using Coherent OTDR," J. Light. Technol. 27, 1142-1146 (2009).

18. L. Chorchos, J. P. Turkiewicz, L. Szostkiewicz, M. Napierala, L. Ostrowski, B. Bienkowska, and T. Nasilowski, "Passive higher order mode filter for 850 nm multimode fiber transmission," Microw. Opt. Technol. Lett. 59, 1959-1962 (2017).

19. X. Lu, M. A. Soto, and L. Thévenaz, "Optimal detection bandwidth for phase-sensitive optical timedomain reflectometry," Sixth European Workshop on Optical Fibre Sensors, Limerick, Ireland, May 2016 